\title{
Coulomb-driven flow of a dielectric liquid subject to charge injection by a sharp electrode
}

\author{
F. J. Higuera ${ }^{a}$ \\ E.T.S. Ingenieros Aeronáuticos, Pza. Cardenal Cisneros 3, 28040 Madrid, Spain \\ P. A. Vázquez \\ Universidad de Sevilla, Depts. Electrónica y Electromagnetismo and Física Aplicada, 41092 Sevilla, Spain
}

(Received 21 January 1999; accepted 23 March 1999)

\begin{abstract}
Injection of charge by a sharp electrode into a surrounding dielectric liquid leads to Coulomb forces that set the liquid into motion. An analysis is presented of this motion in a small region around the edge of the electrode, which determines the injected current as a function of the far electric potential seen by this region. By using an injection law appropriate for nonpolar liquids, the analysis predicts an electric current that increases first exponentially and then as the power $\frac{7}{3}$ of the harmonic part of the electric potential, sometimes with a range of multiplicity in between. (C) 1999 American Institute of Physics. [S1070-6631(99)01207-6]
\end{abstract}

Electric conduction through a purified dielectric liquid between a couple of electrodes subjected to a high potential difference is often dominated by the motion of charge carriers injected at one or the two electrodes by means of electrochemical reactions. In these cases, the presence of a space charge (of density $q$ ) in the electric field $\boldsymbol{E}$ existing in the liquid leads to a Coulomb force $q \boldsymbol{E}$ that induces a motion of the liquid. Such motion enhances charge, momentum and heat transfer, which is of interest in a number of applications including electrostatic precipitators, electrohydrodynamic ion-drag pumps, and electrohydrodynamic laminar and turbulent mixing; see Refs. 1 and 2 for reviews. While strong reproducible injections can be obtained by using electrodialytic membranes, ${ }^{3,4}$ weak and moderate reproducible injections in nonpolar liquids ${ }^{5-7}$ are probably more important in applications. In the first case the charge density at the injecting electrode is nearly independent of the local electric field (autonomous injection), whereas it is a rapidly increasing function of the electric field in the second case. The injection law $q / q_{0}=\left\{\left(E / E_{0}\right)^{1 / 2} K_{1}\left[\left(E / E_{0}\right)^{1 / 2}\right]\right\}^{-1}$, where $E=|\boldsymbol{E}|, K_{1}$ is the modified Bessel function of second kind and order one, and $q_{0}$ and $E_{0}$ are constants, was proposed by Felici and Gosse $^{6}$ on the assumption that the rate limiting process for injection is the extraction of ions through the field-dependent barrier in the so called image-force region on the electrode surface (Schottky effect).

Coulomb-driven convection between plane-parallel electrodes has been much studied; see, e.g., Refs. 8 and 9 and

${ }^{\text {a)} E l e c t r o n i c ~ m a i l: ~ h i g u e r a @ t u p i . d m t . u p m . e s ~}$ references therein. Sharp or peaky electrodes, on the other hand, lead to high electric fields that are of interest, or concern, in applications. Atten and Haidara ${ }^{10}$ determined experimentally the $I-V$ characteristic of a knife-plane electrode assembly in a closed cell, using the high electric field as a means to ascertain the role of charge injection by the sharp electrode in the different regimes found in their experiments, and Takashima et al. ${ }^{11}$ carried out similar experiments and approximate numerical computations for knife-plane and needle-plane electrode configurations. In both cases Coulomb force leads to an electrohydrodynamic plume, analogous to a buoyancy-induced thermal plume, far from the injecting electrode, whose structure and stability have been studied elsewhere (Refs. 12-16). However, it is the flow and charge distribution in a region around the electrode tip of size of the order of its curvature radius that determines the injected current for a given injection law $q=f(E)$ at the electrode surface. This flow was discussed in Ref. 16 and will be further analyzed here under the assumptions that the motion of the liquid is induced locally by Coulomb force, rather than by the recirculation possibly existing in the bulk of the cell, and that the Reynolds number based on the curvature radius of the electrode at its tip and the characteristic velocity of the liquid (see below) is small. Neglecting the inertia of the liquid and the diffusion of the charge, the equations and boundary conditions governing the steady twodimensional flow around a parabolic electrode tip can be written in nondimensional form as follows

$$
\nabla^{2} \psi=-\omega, \quad \text { with } \quad \boldsymbol{v}=(u, v)=\left(\frac{\partial \psi}{\partial y},-\frac{\partial \psi}{\partial x}\right),
$$




$$
\begin{aligned}
& \nabla^{2} \omega+\frac{\partial q}{\partial x} \frac{\partial \phi}{\partial y}-\frac{\partial q}{\partial y} \frac{\partial \phi}{\partial x}=0, \\
& \nabla^{2} \phi=q, \quad \text { with } \quad \boldsymbol{E}=\boldsymbol{\nabla} \phi, \\
& \boldsymbol{\nabla} \cdot[q(\boldsymbol{v}+\kappa \boldsymbol{E})]=0, \\
& x=1-\frac{y^{2}}{4}: \psi=\frac{\partial \psi}{\partial n}=\phi=0, \quad q=f\left(\frac{\partial \phi}{\partial n}\right),
\end{aligned}
$$

and the far field conditions

$$
\left.\begin{array}{l}
\psi \sim \frac{3^{1 / 6}}{2} J^{2 / 3} r^{4 / 3}\left(\cos \frac{2}{3} \theta-\cos \frac{4}{3} \theta\right)+\cdots \\
\omega \sim-\frac{2}{3^{5 / 6}} J^{2 / 3} r^{-2 / 3} \cos \frac{2}{3} \theta+\cdots \\
\phi \sim 3^{1 / 3} J^{1 / 3} r^{2 / 3} \sin \frac{2}{3} \theta+\Phi_{\infty} \phi_{h}+\cdots \\
q=0 \quad \text { at inlet }
\end{array}\right\},
$$

for $(x, y) \rightarrow \infty$. Here $x$ and $y$ are distances along the axis of the parabola and normal thereto, measured from its focus and scaled with twice its curvature radius at the tip $r_{0} / 2 ; r$ $=\left(x^{2}+y^{2}\right)^{1 / 2}$, and $\theta=\arctan (-y / x)$, with $\theta=\pi / 2$ at $x>0$, $y=0$; and $(\psi, \omega, \phi, q)$ are the stream function, the vorticity, the electric potential, and the charge density scaled with $\left(\epsilon E_{c}^{2} r_{0}^{2} / \mu, \epsilon E_{c}^{2} / \mu, E_{c} r_{0}, \epsilon E_{c} / r_{0}\right)$, respectively. Here $\mu$ and $\epsilon$ are the liquid viscosity and permittivity, and $E_{c}$ is a characteristic electric field, depending on the injection law $q$ $=f(E)$ and defined by the condition $f(1)=1$ in nondimensional variables. In what follows the law $q=\exp \{\gamma(E-1)\}$, with $\gamma$ constant, will be used for definiteness, though the results should be valid for any $f(E)$ increasing rapidly with $E$ if $\gamma=d(\ln f) /\left.d E\right|_{E=1}$. Equations (1) and (2) are the mass and momentum conservation equations in the vorticitystream function formulation; the last two terms of Eq. (2) are the curl of the Coulomb force. Equation (3) is Poisson's law for the electric potential and Eq. (4) is the continuity equation for the electric charge, which moves with velocity $\boldsymbol{v}$ $+\kappa \boldsymbol{E}$, where $\kappa$ is the charge mobility nondimensionalized with $\epsilon E_{c} r_{0} / \mu$. The Reynolds number alluded to before is $\mathrm{Re}=\rho \epsilon E_{c}^{2} r_{0}^{2} / \mu^{2}$, and formulation (1)-(6) relies on the assumption $\operatorname{Re} \ll 1$.

The electric field away from the injecting electrode is dominated by the space charge and its image by the electrodes. This charge is confined to a thin streak around the symmetry plane by the action of the converging flow, which in turn is dominated by the Coulomb force acting on the charged streak. The far field conditions (6) result from the balance of the Coulomb force and the viscous shear force on the sides of the streak. The first force is $E_{x} J / u$, where, advancing that $|\boldsymbol{v}| \gg \kappa|\nabla \phi|$ when $x \gg 1, J=\lim _{x \rightarrow \infty} \int_{-\infty}^{\infty} u q d y$ is the electric current per unit depth of the electrode (to be found as part of the solution), so that $J / u$ is the charge density integrated across the streak, and $E_{x}$ is the electric field at the symmetry plane, due mainly to the charged streak and its image by the injecting electrode. This field can be seen to be of order $J / u$. The second force is of order $u / x$, and the balance of the two requires $u=O\left(J^{2 / 3} x^{1 / 3}\right)$ and $E_{x}$ $=O\left(J^{1 / 3} / x^{1 / 3}\right)$. Since $\nabla^{2} \phi=\nabla^{2} \omega=\nabla^{4} \psi=0$ outside the

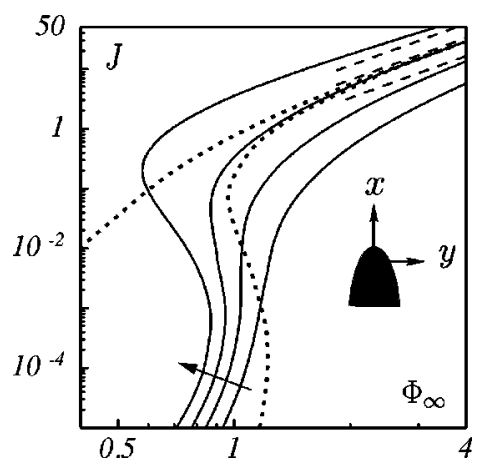

FIG. 1. $J$ as a function of $\Phi_{\infty}$ for $\gamma=20$ and $\kappa=0.2,0.5,1$, and 2, increasing as indicated by the arrow (solid), and for $\kappa=1$ and $\gamma=10$ (dotted left) and $\gamma=30$ (dotted right). The dashed straight lines on the right side are the asymptotes $J=c \kappa^{2 / 3} \Phi_{\infty}^{7 / 3}$ for $\kappa=0.5,1$, and 2, with $c=1.3$.

streak, these asymptotic behaviors lead to the harmonic and biharmonic functions in (6). ${ }^{17}$ In the second term of the asymptotic expansion of $\phi$ in (6) $\phi_{h}=2^{-1 / 2}\left[\left(x^{2}+y^{2}\right)^{1 / 2}\right.$ $+x]^{1 / 2}-1$ is an exact solution of the problem with $q=0$ and the parameter $\Phi_{\infty}$ measures the harmonic field seen by the region of concern. Finally $\partial / \partial n=\left(\partial / \partial x+\frac{1}{2} y \partial / \partial y\right) /(1$ $\left.+y^{2} / 4\right)^{1 / 2}$ denotes the derivative normal to the electrode in (5).

Equations (1)-(4) were rewritten in parabolic coordinates, discretized using second order finite differences, and solved with a pseudotransient method. Values of $\gamma$ ranging from 10 to 30 were used in most of the computations. These values are representative of the limit $\gamma \rightarrow \infty$, in which the extreme sensitivity of the charge density at the electrode with the electric field leads to two regions on the electrode surface: an injection region where $q>0$ and $E=1$, and the rest of the electrode, where $q=0$ and $E<1$. Figure 1 shows $J$ as a function of $\Phi_{\infty}$ for several values of the nondimensional mobility $\kappa$ and of $\gamma$.

As can be seen in Fig. 1, the electric current begins increasing very rapidly with $\Phi_{\infty}$, presents multiplicity in a range of $\Phi_{\infty}$ if $\gamma$ is sufficiently large, and settles to a power law when $\Phi_{\infty} \rightarrow \infty$. On decreasing $J$ in the middle branch, the reduction of the field due to the space charge and its image is nearly balanced by an increase of the harmonic field, so that the field on the electrode decreases very little, as required by the injection law when $\gamma$ is large. (This description and the shape of the curve depend on the manner the two contributions to the field are defined, which is not unique.) In the steep lower branch the harmonic field dominates. The results of Fig. 1 are valid even if the charged streak is nonhomogeneous or unstable beyond the region analyzed here, insofar as the characteristic time of its evolution is large compared with the local residence time $\left(\mu / \epsilon E_{c}^{2}\right.$ in dimensional variables). Then $\Phi_{\infty}$ is a slowly varying function of time and a $S$-shaped response may lead to jumps and hysteresis of the current.

In the absence of space charge, the electric potential is $\Phi_{\infty} \phi_{h}$ and the maximum electric field is $E_{\max }=\Phi_{\infty} / 2$, at the electrode tip, so that the condition $E_{\max }=1$ defining the onset of charge injection for $\gamma \rightarrow \infty$ amounts to $\Phi_{\infty}=2$. This should be the limiting position of the lower turning points in Fig. 1. In addition, since $\phi_{h} \sim x^{1 / 2}$ away from the injecting electrode, 
the onset voltage between the electrodes is $V_{1}=O\left(R^{1 / 2}\right)$, where $R \gg 1$ is the distance between the electrodes scaled with $r_{0}$.

In the asymptotic limit $\Phi_{\infty} \rightarrow \infty$ the charge is confined to a thin layer on the electrode surface and to a streak on the symmetry plane. Let $q$ and $\delta$ be the characteristic charge density in the layer on the electrode and the thickness of this layer, $q_{s}$ and $\delta_{s}$ the corresponding magnitudes in the streak at (nondimensional) distances of order unity from the electrode, and $E=O\left(\Phi_{\infty}\right) \gg 1$ the characteristic electric field. The balance of Coulomb force and viscous force introduced before requires now $E q_{s} \delta_{s} \sim v$, where $v$ is the characteristic velocity of the liquid. This velocity tends to zero at the electrode surface, being of order $v_{\mathrm{cl}}=v \delta$ and essentially tangent to the surface in the charged layer on the electrode, where the velocity normal to the surface is of order $v_{n}=v_{\mathrm{cl}} \delta=v \delta^{2}$ (from the continuity equation). This latter velocity pushes the charge toward the surface against the drift velocity $\kappa E$, which is directed away from the surface. The balance of the two effects determines the thickness of the charged layer, providing the relation $v_{n} \sim \kappa E$, or $q_{s} \delta_{s} \delta^{2} \sim \kappa$. On the other hand, conservation of the injected charge requires $J \sim v q_{s} \delta_{s}$ $\sim v_{\mathrm{cl}} q \delta$, where $q \sim E / \delta$ from Poisson's law in the charged layer [where the electric field must decrease from $O(E)$ in the outer region to essentially $O(1)$ at the surface, specially if $\gamma$ is large]. Thus $q_{s} \delta_{s} \sim(q \delta) v_{\mathrm{cl}} / v \sim E \delta$, and eliminating $q_{s} \delta_{s}$ between this relation and the one coming from the balance of convection and drift velocity yields $\delta \sim(\kappa / E)^{1 / 3}$. Then $q \sim \kappa^{1 / 3} E^{4 / 3}, v \sim \kappa^{1 / 3} E^{5 / 3}$, and $J \sim \kappa^{2 / 3} E^{7 / 3}$. The last result is consistent with the power law relations between the current and the voltage found in some experiments. A comparison with the numerical solution of Eqs. (1)-(6) is presented in Fig. 1, where the dashed lines are $J=c \kappa^{2 / 3} \Phi_{\infty}^{7 / 3}$, with $c=1.3$ chosen to fit the numerical solutions.

The main features of the $J-V$ characteristic of the cell predicted by the present model under stationary conditions and $\gamma \gg 1$ can now be outlined. First, a voltage $V>V_{1}$ $=O\left(R^{1 / 2}\right)$ is required for injection. Second, since the first term of the expansion of $\phi$ in (6) grows with $r$ faster than the second, the excess voltage necessary to keep a stationary current $J$ is $V-V_{1}=O\left(J^{1 / 3} R^{2 / 3}\right)$, so that $J \ll 1$ while $(V$ $\left.-V_{1}\right) \ll R^{2 / 3}$ and $J \gg 1$ when $V \gg R^{2 / 3}$. In the first case the harmonic field dominates in the region $r \ll 1 / J^{2}$ [from (6) with $\left.\Phi_{\infty}=O(1)\right]$, which shrinks when $V$ becomes of $O\left(R^{2 / 3}\right)$. In the second case $\Phi_{\infty}=O\left(J^{3 / 7}\right)$ [taking $\kappa$ $=O(1)]$, which is also large, and the harmonic field dominates in the expanding region $r \ll J^{4 / 7}$. Third, this region ends up covering the whole cell when $J=O\left(R^{7 / 4}\right)$, corresponding to $V=O\left(R^{5 / 4}\right)$, and the relation $J=O\left(V^{3} / R^{2}\right)$ changes to $J$ $=O\left(V^{7 / 3} / R^{7 / 6}\right)$ when $V \gg R^{5 / 4}$. Notice that $\Phi_{\infty}$ $=O\left[\max \left(1, J^{3 / 7}\right)\right]$ is small compared with the value it would have in the absence of space charge, of $O\left(V / R^{1 / 2}\right)$, for any voltage satisfying $V_{1} \ll V \ll R^{5 / 4}$. The space charge is screening the injecting electrode almost completely in this range of voltages. The stability of the stationary flow and the effects of the inertia of the liquid will be analyzed elsewhere.

In summary, an analysis has been carried out of the creeping flow induced by Coulomb forces in a dielectric liq- uid around a sharp two-dimensional electrode injecting charge with a law $q=f(E)$ that reflects the high sensitivity of charge injection with the electric field. The analysis leads to a current-harmonic field relationship $\left(J-\Phi_{\infty}\right)$ that may be monotonically increasing or $S$-shaped, features an injection onset when $\gamma \rightarrow \infty$, and becomes a power law $J \sim \Phi_{\infty}^{7 / 3}$ in the space charge-limited injection limit $\Phi_{\infty} \rightarrow \infty$. An analogous analysis for paraboloidal electrodes leads to the same $\frac{7}{3}$ law up to logarithms.

\section{ACKNOWLEDGMENTS}

We are grateful to Profs. A. Castellanos and A. T. Pérez for useful discussions. This work was supported by DGICYT grant PB95-0008.

${ }^{1}$ A. Castellanos, "Coulomb-driven convection in electrohydrodynamics," IEEE Trans. Electr. Insul. 26, 1201 (1991).

${ }^{2} \mathrm{P}$. Atten, "Electrohydrodynamic instability and motion induced by injected space charge in insulating liquids,' IEEE Trans. Dielectr. Electr. Insul. 3, 1 (1996).

${ }^{3}$ G. Brière and J. P. Gosse, “Injection d'ions dans les liquides polaires,' J. Chim. Phys. 65, 725 (1968).

${ }^{4}$ N. J. Felici and R. E. Tobazéon, "Charge carrier elimination and production by electro-dialytic polymers in contact with dielectric liquids,' J. Electrost. 11, 135 (1981).

${ }^{5}$ A. Denat, B. Gosse, and J. P. Gosse, "Ion injections in hydrocarbons,', J. Electrost. 7, 205 (1979).

${ }^{6}$ N. J. Felici and J. P. Gosse, "Injection d'ions par des électrodes métaliques dans les hydrocarbures liquides de résistivité éleveé,' Rev. Phys. Appl. 14, 629 (1979).

${ }^{7}$ A. Alj, A. Denat, J. P. Gosse, B. Gosse, and I. Nakamura, "Creation of charge carriers in nonpolar liquids" IEEE Trans. Electr. Insul. 20, 221 (1985).

${ }^{8}$ J. M. Schneider and P. K. Watson, "Electrohydrodynamic stability of space-charge-limited currents in dielectric liquids. I. Theoretical study,', Phys. Fluids 13, 1948 (1970).

${ }^{9}$ R. Chicón, A. Castellanos, and E. Martín, "Numerical modelling of Coulomb-driven convection in insulating liquids,', J. Fluid Mech. 344, 43 (1997).

${ }^{10} \mathrm{P}$. Atten and M. Haidara, "Electrical conduction and EHD motion of dielectric liquids in a knife-plate electrode assembly,', IEEE Trans. Electr. Insul. 20, 187 (1985).

${ }^{11} \mathrm{~T}$. Takashima, R. Hanaoka, R. Ishibashi, and A. Ohtsubo, " $I-V$ characteristics and liquid motion in needle-to-plane and razor-blade-to-plane configurations in transformer oil and liquid nitrogen,' IEEE Trans. Electr. Insul. 23, 645 (1988).

${ }^{12}$ F. M. J. McCluskey and A. T. Pérez, “The electrohydrodynamic plume between a line source of ions and a flat plate,', IEEE Trans. Electr. Insul. 27, 334 (1992).

${ }^{13} \mathrm{P}$. Atten, B. Malraison, and M. Zahn, "Electrohydrodynamic plumes in point-plane geometry,' IEEE 11th ICDL, Baden, Switzerland (1993).

${ }^{14}$ A. T. Pérez, P. A. Vázquez, and A. Castellanos, "Dynamics and linear stability of charged jets in dielectric liquids,' IEEE Trans. Ind. Appl. 31, 761 (1995).

${ }^{15}$ A. T. Pérez, P. A. Vázquez, and A. Castellanos, “Thermal and electrohydrodynamical plumes. A comparative study,', Phys. Fluids 8, 2091 (1996).

${ }^{16}$ P. A. Vázquez, "Dinámica y estabilidad de penachos electrohidrodinámicos," Doctor Thesis, Universidad de Sevilla, Spain (1998).

${ }^{17}$ The scaling and the boundary conditions at the electrode $(\theta \approx 0)$ imply $\phi \sim A r^{2 / 3} \sin \frac{2}{3} \theta$ and $\psi \sim B r^{4 / 3}\left(\cos \frac{2}{3} \theta-\cos \frac{4}{3} \theta\right)$, whence $E_{x}=\partial \phi /\left.\partial r\right|_{\theta=\pi}$ $=3^{-1 / 2} A / r^{1 / 3}, u=r^{-1} \partial \psi /\left.\partial \theta\right|_{\theta=\pi}=3^{1 / 2} B r^{1 / 3}$, and the viscous stress on the sides of the streak is $2 r^{-2} \partial^{2} \psi /\left.\partial \theta^{2}\right|_{\theta=\pi}=\frac{4}{3} B / r^{2 / 3}$. On the other hand, using the expression $J / u=J /\left(3^{1 / 2} B r^{1 / 3}\right)$ for the charge in the streak, electrostatics gives $E_{x}=J /\left(2 B r^{1 / 3}\right)$ and the Coulomb force $E_{x} J / u=J^{2} /(2$ $\left.\times 3{ }^{1 / 2} B^{2} r^{2 / 3}\right)$. The constants $B$ and $A$ used in (6) then follow by equating this force to the viscous force and by equating the two expressions of $E_{x}$ above. 